\title{
Discontinuous Irregular Oblique Derivative Problems for Nonlinear Elliptic Equations of Second Order
}

\author{
Guochun Wen and Zuoliang Xu
}

\begin{abstract}
In this paper, the discontinuous irregular oblique derivative problems (or discontinuous Poincaré boundary value problems) for nonlinear elliptic equations of second order in multiply connected domains are discussed by using a complex analytic method. Firstly the uniqueness of solutions for such boundary value problems is proved and a priori estimates of their solutions are given, and then by the LeraySchauder theorem, the existence of solutions of the above problems is verified. As a special case the result about the continuous irregular oblique derivative problem for the nonlinear equations is derived.
\end{abstract}

Keywords. Nonlinear elliptic equations, discontinuous irregular oblique derivative problems, complex analytic method

Mathematics Subject Classification (2000). Primary 35J65, secondary 35J60

\section{Formulation of the problem}

It is known that there are many problems in mechanics and physics, whose boundary conditions are discontinuous (see $[1,3]$ ). In $[1,2], \mathrm{H}$. Begehr and G. C. Wen studied discontinuous Riemann-Hilbert problem and oblique derivative problem for nonlinear elliptic complex equations, and proved the existence and uniqueness of these boundary value problems. In [3], V. N. Monakhov considered the Poincaré problem with discontinuous coefficients in the boundary condition for a quasilinear second order elliptic equation by using function theoretic methods. This paper deals with the discontinuous irregular oblique

Guochun Wen: School of Mathematical Sciences, Peking University, Beijing 100871, China; wengc@math.pku.edu.cn

Zuoliang Xu: School of Information, Renmin University of China, Beijing 100872, China; xuzl@ruc.edu.cn

This research is supported by the National Natural Science Foundation of China (10671207) and China Postdoctoral Science Foundation (2005037447) 
derivative problems for nonlinear elliptic equations of second order. The results in this paper are further developments of the theory of nonlinear elliptic complex equations as stated in [1-7].

Let $D$ be an $(N+1)$-connected bounded domain in the complex plane $\mathbb{C}$ with the boundary $\Gamma=\bigcup_{j=0}^{N} \Gamma_{j} \in C_{\mu}^{2}(0<\mu<1)$, where $\Gamma_{j}(j=1, \ldots, N)$ are located in the domain $D_{0}=\{|z|<1\}$ bounded by $\Gamma_{0}=\Gamma_{N+1}$, and $0 \in D$. Without loss of generality, we can assume that $D$ is a circular domain in $|z|<1$ with the boundary $\Gamma_{N+1}=\{|z|=1\}$ and $\Gamma_{j}=\left\{\left|z-a_{j}\right|=r_{j}\right\}, j=1, \ldots, N$. Introduce the second order nonlinear elliptic equation in the complex form (see [6])

$$
\left\{\begin{aligned}
u_{z \bar{z}} & =F\left(z, u, u_{z}, u_{z z}\right) \\
F & =\operatorname{Re}\left[Q u_{z z}+A_{1} u_{z}\right]+\hat{A}_{2} u+A_{3} \\
Q & =Q\left(z, u, u_{z}, u_{z z}\right) \\
A_{j} & =A_{j}\left(z, u, u_{z}\right), \quad j=1,2,3 \\
\hat{A}_{2} & =A_{2}+|u|^{\sigma},
\end{aligned}\right.
$$

where $\sigma$ is a positive number. Moreover we consider the equation of second order

$$
u_{x x}+b(u) u_{x y}+u_{y y}-|u|^{\sigma} u=0 \text { in } D
$$

with the coefficient $b(u)=u^{2}$ if $|u| \leq 1$ and $b(u)=u^{-2}$ if $|u|>1$, whose complex form is

$$
u_{z \bar{z}}=\operatorname{Re}\left[Q u_{z z}\right]+\hat{A}_{2} u \text { in } D
$$

where $Q=Q(u)=-\frac{1}{2} i b(u), \hat{A}_{2}=\frac{1}{4}|u|^{\sigma}$ with the condition $|Q| \leq \frac{1}{2}<1$. It is clear that the above complex equation is a special case of (1).

Suppose that (1) satisfies the following

\section{Conditions $C^{*}$ :}

1) $Q\left(z, u, u_{z}, U\right), A_{j}\left(z, u, u_{z}\right), j=1,2,3$, are continuous in $u \in \mathbb{R}, u_{z} \in \mathbb{C}$ for almost every $z \in D, U \in \mathbb{C}$, and $Q=0, A_{j}=0, j=1,2,3$, for $z \notin D$.

2) The above functions are measurable in $z \in D$ for any continuously differentiable function $u(z)$ in $D^{*}$ and any measurable function $U(z) \in L_{p_{0}}(\tilde{D})$, and satisfy

$$
L_{p}\left[A_{1}\left(z, u, u_{z}\right), \bar{D}\right] \leq k_{0}, L_{p}\left[A_{2}\left(z, u, u_{z}\right), \bar{D}\right] \leq k_{0}, L_{p}\left[A_{3}\left(z, u, u_{z}\right), \bar{D}\right] \leq k_{1} .
$$

Here $p_{0}, p\left(2<p_{0} \leq p\right), k_{0}, k_{1}$ are non-negative constants, and $D^{*}=\bar{D} \backslash T$, $T=\left\{z_{1}, \ldots, z_{m+1}\right\}, z_{1}, \ldots, z_{m+1}$ (or $z_{K^{\prime}}, K^{\prime}$ is an integer as stated in (2) below) are distinct points arranged according to the positive direction on $\Gamma, \tilde{D}$ is any closed subdomain in $D^{*}$. 
3) The equation (1) satisfies the uniform ellipticity condition

$$
\left|F\left(z, u, u_{z}, U_{1}\right)-F\left(z, u, u_{z}, U_{2}\right)\right| \leq q_{0}\left|U_{1}-U_{2}\right|, \quad \hat{A}_{2} \geq 0 \text { in } D,
$$

for almost every point $z \in D$, any continuously differentiable function $u(z)$ in $D^{*}$ and $U_{1}, U_{2} \in \mathbb{C}$, where $q_{0}<1$ is a non-negative constant. We can assume that there exists at least one point of discontinuity on $\Gamma_{j}(1 \leq j \leq N+1)$. Because for any component $\Gamma_{j}(1 \leq j \leq N+1)$ if $\lambda(z)$ on such component is continuous, we can choose a point on this component as a point of discontinuity. It is obvious that in this case the partial index $K_{j}$ of $\lambda(z)$ on $\Gamma_{j}(1 \leq j \leq N+1)$ is unchanged. Denote by $\Gamma_{k}^{\prime}$ the curve on $\Gamma$ from $z_{k-1}$ to $z_{k}$ (if there is only one point of discontinuity on $\Gamma_{j}(1 \leq j \leq N+1)$, then $\left.z_{k}=z_{k-1}(1 \leq k \leq m+1)\right)$, and in general $\Gamma_{k}^{\prime}$ includes the initial point $z_{k-1}$ and does not include the terminal point $z_{k}(k=2, \ldots, m+2)$.

Problem $P$. Find a continuous solution $u(z)$ of $(1)$ in $\bar{D}$, whose partial derivatives $u_{x}, u_{y}$ in $D^{*}=\bar{D} \backslash T$ are continuous, and satisfies the boundary conditions

$$
\frac{\partial u}{\partial \nu}+2 a_{1}(z) u(z)=2 a_{2}(z), \quad z \in \Gamma^{*}=\Gamma \backslash T, u\left(z_{k}\right)=b_{k}, \quad k=0,1, \ldots, K^{\prime},
$$

where $\nu\left(=\nu_{1}+i \nu_{2} \neq 0\right)$ can be arbitrary at every point on $\Gamma^{*}, K^{\prime}\left(\geq J^{\prime}\right)$ is a non-negative integer, $z_{0} \in \Gamma_{J+1}$, the numbers $J, J^{\prime}(\geq 0)$ will be stated below. It is easy to see that the boundary condition (2) can be rewritten in the complex form

$$
\operatorname{Re}\left[\overline{\lambda(z)} u_{z}\right]+a_{1}(z) u=a_{2}(z), \quad z \in \Gamma^{*}, \quad u\left(z_{k}\right)=b_{k}, \quad k=0,1, \ldots, K^{\prime},
$$

in which $\lambda(z)=\cos (\nu, x)+i \sin (\nu, x)=e^{i(\nu, x)} \neq 0,(\nu, x)$ is the angle between $\nu$ and the $x$-axis. It is easy to see that $u\left(z_{k}\right)=b_{k}\left(k=0,1, \ldots, K^{\prime}\right)$ are definite, because $u(z)$ is continuous in $\bar{D}$ and $u_{x}, u_{y}$ or $u_{z}$ at $z_{j}\left(j=0,1, \ldots, K^{\prime}\right)$ maybe the points of discontinuity. Suppose that $\lambda(z), a_{1}(z), a_{2}(z)$ satisfy the conditions

$$
\begin{aligned}
& C_{\alpha}\left[\lambda(z), \Gamma_{k}^{\prime}\right] \leq k_{0}, \quad\left|b_{k}\right| \leq k_{2}, \quad k=0,1, \ldots, K^{\prime}, \\
& C_{\alpha}\left[a_{1}(z), \Gamma_{k}^{\prime}\right] \leq k_{0}, \quad 0 \leq a_{1}(z) \cos (\nu, n) \text { on } \Gamma^{*}=\Gamma \backslash T, \\
& C_{\alpha}\left[R_{k}(z) a_{2}(z), \Gamma_{k}^{\prime}\right] \leq k_{2}, \quad k=1, \ldots, m+1,
\end{aligned}
$$

in which $n$ is the outward normal vector on $\Gamma^{*}$ and

$$
R_{k}(z)=\left|z-z_{k-1}\right|^{\beta_{k-1}}\left|z-z_{k}\right|^{\beta_{k}},
$$

$\beta_{k}<1(k=1, \ldots, m+1)$, and $\alpha\left(\frac{1}{2}<\alpha<1\right), k_{2}$ are non-negative constants.

For convenience, we assume that $\cos (\nu, n) \equiv 0, a_{1}(z) \equiv 0$ on $\Gamma_{k}^{\prime}(k=1, . ., J)$, and the above identical formulas on $\Gamma_{k}^{\prime}(J+1 \leq k \leq m+1)$ do not hold. But if $L$ is the longest curve on $\Gamma_{k}^{\prime}(J+1 \leq k \leq m+1)$ such that $\cos (\nu, n)=0$, $a_{1}(z)=0$, then we require that it appears one of the following four cases: 
1. One end point of $L$ belongs to $\tilde{T}=\left\{z_{0}, z_{1}, \ldots, z_{K^{\prime}}\right\}$ and $\int_{\Gamma_{j}} a_{2}(z) d s=0$, if $\cos (\nu, n)=0, a_{1}(z)=0$ on $\Gamma_{j}, 1 \leq j \leq J^{\prime}(\leq N+1)$.

2. The inequality $\cos (\nu, n) \cos (\nu, s)>0$ in the neighborhood of the initial point of $L$ on $\Gamma_{k}^{\prime} \backslash L$ holds.

3. The inequality $\cos (\nu, n) \cos (\nu, s)<0$ in the neighborhood of the terminal point of $L$ on $\Gamma_{k}^{\prime} \backslash L$ holds, where $s$ is the tangent vector of $\Gamma_{k}^{\prime}$ identical to the positive direction of $\Gamma$, and $\Gamma^{\prime}=\bigcup_{k=1}^{J} \Gamma_{k}^{\prime}, \Gamma^{\prime \prime}=\bigcup_{k=J+1}^{m+1} \Gamma_{k}^{\prime}$.

4. If the initial and terminal points of $L$ do not conform to the conditions in cases 2 and 3 , then there is one point condition which belongs to $\tilde{T}$. Problem $P$ with homogeneous boundary condition of $(2)$ or $(3)$ is called Problem $P_{0}$.

The number $K=\frac{1}{2}\left(K_{1}+\cdots+K_{m+1}\right)$ is called the index of Problem $P$, where

$$
\begin{aligned}
K_{k} & =\left[\frac{\phi_{k}}{\pi}\right]+J_{k}, \quad J_{k}=0 \text { or } 1, \\
e^{i \phi_{k}} & =\frac{\lambda\left(z_{k}-0\right)}{\lambda\left(z_{k}+0\right)}, \quad \gamma_{k}=\frac{\phi_{k}}{\pi}-K_{k}, \quad k=1, \ldots, m+1 .
\end{aligned}
$$

Here we choose the index $K \geq N-\frac{1}{2}$ and denote $K^{\prime}=2 K-2 N+J^{\prime}+1$.

Problem $Q$. In addition we consider the discontinuous boundary value problem: Equation (1) and boundary condition (2) are replaced by

$$
\left\{\begin{aligned}
u_{z \bar{z}} & =G\left(z, u, u_{z}, u_{z z}\right) \\
G & =\operatorname{Re}\left[Q u_{z z}+A_{1} u_{z}\right]+A_{3} \\
Q & =Q\left(z, u, u_{z}, u_{z z}\right) \\
A_{j} & =A_{j}\left(z, u, u_{z}\right), \quad j=1,3 .
\end{aligned}\right.
$$

and

$$
\begin{aligned}
\operatorname{Re}\left[\overline{\lambda(z)} u_{z}\right] & =a_{2}(z), \quad z \in \Gamma^{*} \\
u\left(z_{0}\right) & =b_{0} \\
\operatorname{Im}\left[\overline{\lambda(z)} u_{z}\right]_{z=z_{k}^{\prime}} & =b_{k}^{\prime}, \quad k=1, \ldots, K^{\prime},
\end{aligned}
$$

respectively, in which $z_{k}^{\prime} \notin T\left(k=1, \ldots, K^{\prime}\right)$ are distinct points on $\Gamma^{*}$ as stated in $[6], b_{k}^{\prime}\left(k=1, \ldots, K^{\prime}\right)$ are real constants satisfying the conditions $\left|b_{k}^{\prime}\right| \leq k_{2}, \quad k=1, \ldots, K^{\prime}$, such that the above boundary value problem is well-posed. The above boundary value problem with homogeneous boundary condition is called Problem $Q_{0}$.

It is clear that Problem $P$ is a discontinuous irregular oblique derivative problem. If $\cos (\nu, n)>0$ on $\Gamma$, then Problem $P$ is the discontinuous third boundary value problem (Problem III or $O$ ). If $\cos (\nu, n)=1, a_{1}(z)=0$ on $\Gamma$, then Problem $P$ is the discontinuous Neumann boundary problem (Problem II). If $\cos (\nu, n)=0, a_{2}(z) \equiv 0$ on $\Gamma$, Problem $P$ is equivalent to the discontinuous first boundary value problem (Problem I). 


\section{Uniqueness and estimates of solutions}

First of all we prove the following result.

Theorem 2.1. Suppose that equation (1) satisfies Conditions $C^{*}$. Then Problem $P_{0}$ for equation (1) with the condition $A_{3}=0$ in $D$ has only the trivial solution.

Proof. Let $u(z)$ be any solution of Problem $P_{0}$ for equation (1) with $A_{3}=0$. From Conditions $C^{*}$, it is easily seen that $u(z)$ is a solution of the uniformly elliptic equation:

$$
u_{z \bar{z}}=\operatorname{Re}\left[Q u_{z z}+A_{1} u_{z}\right]+\hat{A}_{2} u, \quad|Q| \leq q_{0}<1, \quad \hat{A}_{2}=A_{2}+|u|^{\sigma} \geq 0 \text { in } D
$$

and satisfies the boundary conditions

$$
\frac{\partial u}{\partial \nu}+2 a_{1}(z) u(z)=0 \text { on } \Gamma^{*}, \quad u\left(z_{j}\right)=0, \quad j=0,1, \ldots, K^{\prime}
$$

Substitute the solution $u(z)$ into the coefficients of equation (7), we can find a solution $\Psi(z)$ of $(7)$ satisfying the condition $\Psi(z)=1$ on $\Gamma$. Thus the function $U(z)=\frac{u(z)}{\Psi(z)}$ is a solution of the equation

$$
U_{z \bar{z}}=\operatorname{Re}\left[Q U_{z z}+A_{0} U_{z}\right], \quad A_{0}=-2(\log \Psi)_{\bar{z}}+2 Q(\log \Psi)_{z}+A_{1},
$$

satisfying the boundary conditions

$$
\frac{\partial U}{\partial \nu}+a_{1}^{*}(z) U(z)=0 \text { on } \Gamma^{*}, \quad U\left(z_{j}\right)=0, \quad j=0,1, \ldots, K^{\prime},
$$

where $a_{1}^{*}(z)=a_{1}(z)+\frac{(\partial \Psi / \partial \nu)}{\Psi(z)}, a_{1}^{*}(z) \cos (\nu, n) \geq 0$ on $\Gamma^{*}$. If $M=\max _{\bar{D}} U(z)>0$ in $D$, then there exists a point $z^{*} \in \Gamma$ such that $M=U\left(z^{*}\right)=\max _{\bar{D}} U(z)>0$. For the case 1 , when $z^{*} \in \Gamma^{\prime}$, noting that $\cos (\nu, n) \equiv 0, a_{1}(z) \equiv 0, \frac{\partial \Psi(z)}{\partial \nu} \equiv 0$ on $\Gamma^{\prime}$, we have $\frac{\partial U}{\partial \nu} \equiv 0, U(z) \equiv M$ on $\Gamma_{j}\left(1 \leq j \leq J^{\prime}\right)$. This contradicts the point conditions in (8). For the cases 2 and 3 , when $z^{*} \in \Gamma^{\prime \prime}$, if $\cos (\nu, n)>0$ at $z^{*}$, from [6, Chap. III, Cor. 2.10], we have $\frac{\partial U}{\partial \nu}>0$ at $z^{*}$. This contradicts the condition (8) on $\Gamma^{\prime \prime}$. If $\cos (\nu, n)=0$ and $a_{1}^{*}\left(z^{*}\right) \neq 0$ at $z^{*}$, then $\frac{\partial U}{\partial \nu}+a_{1}^{*}(z) U \neq 0$ at $z^{*}$. This is also impossible. Denote by $L$ the longest curve of $\Gamma$ including the point $z^{*}$, such that $\cos (\nu, n)=0$ and $a^{*}(z)=0$, thus $u(z)=M$ on $L$. From the point conditions in (8), any point of $\tilde{T}=\left\{z_{0}, z_{1}, \ldots, z_{K^{\prime}}\right\}$ cannot be an end point of $L$, then there exists a point $z^{\prime} \in \Gamma^{\prime \prime} \backslash\{L \cup \tilde{T}\}$, such that at $z^{\prime}$, $\cos (\nu, n)>0(<0), \frac{\partial U}{\partial n}>0, \cos (\nu, s)>0(<0), \frac{\partial U}{\partial s} \geq 0$, or $\cos (\nu, n)<0(>0)$, $\frac{\partial U}{\partial n}>0, \cos (\nu, s)>0(<0), \frac{\partial U}{\partial s} \leq 0$. Hence

$$
\frac{\partial U}{\partial \nu}=\cos (\nu, n) \frac{\partial U}{\partial n}+\cos (\nu, s) \frac{\partial U}{\partial s}>0, \quad \text { or }<0 \text { at } z^{\prime}
$$


holds, where $s$ is the tangent vector at $z^{\prime} \in \Gamma^{\prime \prime}$ identical to the positive direction of $\Gamma$, and then

$$
\frac{\partial U}{\partial \nu}+a_{1}^{*} U>0, \quad \text { or } \frac{\partial U}{\partial \nu}+a_{1}^{*} U<0 \text { at } z^{\prime}
$$

it is also impossible. For the case 4 , when $z^{*} \in \Gamma^{\prime \prime}$, it will be occurred the similar case as in the case 1 . This shows that $u(z)$ cannot attain its maximum $M$ at a point $z^{*} \in \Gamma$. Similarly we can prove that $u(z)$ cannot attain its minimum at a point $z_{*} \in \Gamma$, hence $u(z)=0$ on $\Gamma$, thus $u(z)=0$ in $\bar{D}$.

By a similar way as stated before, we can prove the uniqueness theorem of solutions of Problem $P$ for equation (1) with $\sigma=0$ as follows.

Corollary 2.2. Suppose that equation (1) with $\sigma=0$ satisfies Conditions $C^{*}$ and the following condition: for any real functions $u_{j}(z) \in C^{1}\left(D^{*}\right), V_{j}(z) \in$ $L_{p_{0}}(\tilde{D})(j=1,2)$, the equality

$$
\begin{aligned}
& F\left(z, u_{1}, u_{1 z}, V_{1}\right)-F\left(z, u_{2}, u_{2 z}, V_{2}\right)= \\
& \operatorname{Re}\left[\tilde{Q}\left(V_{1}-V_{2}\right)+\tilde{A}_{1}\left(u_{1}-u_{2}\right)_{z}\right]+\tilde{A}_{2}\left(u_{1}-u_{2}\right) \text { in } D
\end{aligned}
$$

holds, where $|\tilde{Q}| \leq q_{0}$ in $D, \tilde{A}_{1}, \tilde{A}_{2} \in L_{p_{0}}(\bar{D})$. Then Problem $P_{0}$ for equation (1) has at most one solution.

Theorem 2.3. Let equation (1) satisfy Conditions $C^{*}$. Then any solution of Problem $P$ for (1) satisfies the estimates

$$
\begin{aligned}
\hat{C}_{\delta}^{1}[u, \bar{D}] & =C_{\delta}\left[|u|^{\sigma+1}, \bar{D}\right]+C_{\delta}\left[X(z) u_{z}, \bar{D}\right] \leq M_{1} \\
\|u\|_{\tilde{W}_{p_{0}}^{2}(D)} & =\left\|X(z) u_{z}\right\|_{W_{p_{0}}^{1}(D)} \leq M_{1}, \hat{C}_{\delta}^{1}[u, \bar{D}] \leq M_{2}\left(k_{1}+k_{2}\right),
\end{aligned}
$$

where $X(z)$ is given as

$$
X(z)=\prod_{j=1}^{m+1}\left|z-z_{j}\right|^{\eta_{j}}, \quad \eta_{j}=\left\{\begin{array}{l}
\left|\gamma_{j}\right|+\tau, \text { if } \gamma_{j}<0, \beta_{j} \geq\left|\gamma_{j}\right| \\
\left|\beta_{j}\right|+\tau, \text { if } \gamma_{j} \geq 0, \text { or } \gamma_{j}<0, \beta_{j}<\left|\gamma_{j}\right| .
\end{array}\right.
$$

Here $\gamma_{j}(j=1, \ldots, m+1)$ are real constants as stated in $(5), \tau, \delta$ (with $\delta \leq$ $\min [\beta, \tau], 0<\beta \leq \alpha)$ are sufficiently small positive constants, $k=\left(k_{0}, k_{1}, k_{2}\right)$, $M_{1}=M_{1}\left(q_{0}, p_{0}, \alpha, k, p, D\right), M_{2}=M_{2}\left(q_{0}, p_{0}, \alpha, k_{0}, p, D\right)$ are non-negative constants.

Proof. By using the reduction to absurdity, we shall prove that any solution $u(z)$ of Problem $P$ satisfies the estimate of boundedness

$$
\hat{C}^{1}[u, \bar{D}]=C\left[|u|^{\sigma+1}, \bar{D}\right]+C\left[X(z) u_{z}, \bar{D}\right] \leq M_{3},
$$


where $M_{3}=M_{3}\left(q_{0}, p_{0}, \alpha, k, p, D\right)$ is a non-negative constant. Suppose that (12) is not true, then there exist sequences of coefficients $\left\{A_{j}^{(m)}\right\}(j=1,2,3),\left\{Q^{(m)}\right\}$, $\left\{\lambda^{(m)}(z)\right\},\left\{a_{j}^{(m)}\right\}(j=1,2), b_{j}^{(m)}\left(j=0,1, \ldots, K^{\prime}\right)$, which satisfy Conditions $C^{*}$ and (4), such that $\left\{A_{j}^{(m)}\right\}(j=1,2,3),\left\{Q^{(m)}\right\},\left\{\lambda^{(m)}(z)\right\},\left\{a_{j}^{(m)}\right\}(j=1,2)$ and $\left\{b_{j}^{(m)}\right\}\left(j=0,1, \ldots, K^{\prime}\right)$ in $\bar{D}, \Gamma$ weakly converge or uniformly converge to $A_{j}^{(0)}$ $(j=1,2,3), Q^{(0)}, \lambda^{(0)}(z), a_{j}^{(0)}(j=1,2), b_{j}^{(0)}\left(j=0,1, \ldots, K^{\prime}\right)$, respectively. Moreover the corresponding boundary value problems

$$
u_{z \bar{z}}-\operatorname{Re}\left[Q^{(m)} u_{z z}+A_{1}^{(m)} u_{z}\right]-\hat{A}_{2}^{(m)} u=A_{3}^{(m)}, \quad \hat{A}_{2}^{(m)}=A_{2}^{(m)}+|u|^{\sigma},
$$

and

$$
\frac{\partial u}{\partial \nu}+2 a_{1}^{(m)}(z) u=2 a_{2}^{(m)}(z) \text { on } \Gamma^{*}, \quad u\left(z_{j}\right)=b_{j}^{(m)}, \quad j=0,1, \ldots, K^{\prime},
$$

have the solutions $\left\{u^{(m)}(z)\right\}$ with unbounded $\hat{C}\left[u^{(m)}(z), \bar{D}\right](m=1,2, \ldots)$. We can choose a subsequence of $\left\{u^{(m)}(z)\right\}$ denoted by $\left\{u^{(m)}(z)\right\}$ again, such that $h_{m}=\hat{C}\left[u^{(m)}(z), \bar{D}\right] \rightarrow \infty$ as $m \rightarrow \infty$, and assume that $h_{m} \geq \max \left[k_{1}, k_{2}, 1\right]$. It is obvious that $\tilde{u}^{(m)}(z)=\frac{u^{(m)}(z)}{h_{m}}(m=1,2, \ldots)$ are solutions of the boundary value problems

$$
\tilde{u}_{z \bar{z}}-\operatorname{Re}\left[Q^{(m)} \tilde{u}_{z z}+A_{1}^{(m)} \tilde{u}_{z}\right]-\hat{A}_{2}^{(m)} \tilde{u}=\frac{A_{3}^{(m)}}{h_{m}}
$$

and

$$
\frac{\partial \tilde{u}}{\partial \nu}+2 a_{1}^{(m)}(z) \tilde{u}=\frac{2 a_{2}^{(m)}(z)}{h_{m}} \text { on } \Gamma^{*}, \quad \tilde{u}\left(z_{j}\right)=\frac{b_{j}^{(m)}}{h_{m}}, j=0,1, \ldots, K^{\prime} .
$$

We can see that the functions in the above equation and the boundary conditions satisfy the Conditions $C^{*},(4)$, and

$$
\begin{array}{rlrl}
\frac{|\tilde{u}|^{\sigma+1}}{h_{m}} & \leq 1, \quad L_{\infty}\left[\frac{A_{3}^{(m)}}{h_{m}}, \bar{D}\right] \leq 1, \\
\left|\frac{a_{2}^{(m)}}{h_{m}}\right| & \leq 1, & \left|\frac{b_{j}^{(m)}}{h_{m}}\right| & \leq 1, \quad j=0,1, \ldots, K^{\prime} .
\end{array}
$$

Hence from [6, Chap. III, Thm. 10], we can obtain the estimate

$$
\hat{C}_{\delta}^{1}\left[\tilde{u}^{(m)}(z), \bar{D}\right] \leq M_{4}, \quad\left\|X(z) \tilde{u}_{z}^{(m)}\right\|_{\tilde{W}_{p_{0}}^{1}(D)} \leq M_{4},
$$

in which $M_{4}=M_{4}\left(q_{0}, p_{0}, \beta, k, D\right)$ is a non-negative constant. Thus from the sequence of functions $\left\{\tilde{u}^{(m)}(z)\right\}$, we can choose a subsequence denoted by $\left\{\tilde{u}^{(m)}(z)\right\}$ again, which in $\bar{D}$ uniformly converges to $\tilde{u}_{0}(z)$, and $X(z) \tilde{u}_{x}^{(m)}, X(z) \tilde{u}_{y}^{(m)}$ in $\bar{D}$ 
are uniformly convergent and $\tilde{u}_{x x}^{(m)}, \tilde{u}_{y y}^{(m)}, \tilde{u}_{x y}^{(m)}$ in $\tilde{D}$ are weakly convergent. This shows that $\tilde{u}_{0}(z)$ is a solution of the boundary value problem

$$
\tilde{u}_{0 z \bar{z}}-\operatorname{Re}\left[Q^{(0)} \tilde{u}_{0 z z}+A_{1}^{(0)} \tilde{u}_{0 z}\right]-\hat{A}_{2}^{(0)} \tilde{u}_{0}=0,
$$

and

$$
\frac{\partial \tilde{u}_{0}}{\partial \nu}+2 a_{1}^{(0)}(z) \tilde{u}_{0}=0 \text { on } \Gamma^{*}, \quad u_{0}\left(z_{j}\right)=0, \quad j=0,1, \ldots, K^{\prime} .
$$

We see that the coefficient $A_{3}^{(0)}$ in equation (19) is equal to 0 and boundary condition (20) is homogeneous. On the basis of Theorem 2.1, the solution $\tilde{u}_{0}(z)=0$, however, from $\hat{C}^{1}\left[\tilde{u}^{(m)}(z), \bar{D}\right]=1$, we can derive that there exists a point $z^{*} \in \bar{D}$, such that $\left.\left[\left|\tilde{u}_{0}(z)\right|^{\sigma+1}+\left|X(z) u_{z}\right|\right]\right|_{z=z^{*}} \neq 0$. This is impossible. This shows that the first two estimates in (10) are true. Moreover it is not difficult to verify the third estimate in (10).

\section{Solvability}

In this section, we mainly prove the solvability of Problem $P$ for equation (24) below. In the following we first discuss the solution of equation (6).

Theorem 3.1. Let equation (1) in D satisfy Conditions $C^{*}$. Then Problems $P$ and $Q$ for equation (6) are solvable, and the solution $u(z)$ satisfies

$$
\hat{C}_{\delta}^{1}[u, \bar{D}]=C_{\delta}\left[|u|^{\sigma+1}, \bar{D}\right]+C_{\delta}[X(z) w(z), \bar{D}] \leq M_{5},
$$

in which $M_{5}=M_{5}\left(p_{0}, \delta, k, D\right)$ is a non-negative constant, and $X(z)$ is as stated in (11). Besides the solution of Problems $P$ and $Q$ for (6) are unique, if the condition (9) holds.

Proof. It is clear that Problem $Q$ for (6) is equivalent to Problem $A$ for the first order complex equation

$$
w_{\bar{z}}=\operatorname{Re}\left[Q w_{z}+A_{1} w\right]+A_{3}
$$

with the boundary conditions

$$
\begin{aligned}
\operatorname{Re}[\overline{\lambda(z)} w(z)] & =a_{2}(z), \quad z \in \Gamma^{*} \\
u\left(z_{0}\right) & =b_{0} \\
\operatorname{Im}\left[\overline{\lambda\left(z_{j}^{\prime}\right)} w\left(z_{j}^{\prime}\right)\right] & =b_{j}^{\prime}, \quad j=1, \ldots, K^{\prime},
\end{aligned}
$$

and the relation

$$
u(z)=2 \operatorname{Re} \int_{z_{0}}^{z} w(z) d z+b_{0} \text { in } D
$$


(see [6, Chap. 6]). From Theorems 2.3 and 2.4 [6, Chap. IV], we see that Problem $A$ for $(22)$ has a solution $w(z)$ satisfying the estimate (21), and $u(z)$ in (23) is the solution of Problem $Q$ for $(22)$. Next let $u_{0}(z)$ be a solution of Problem $Q$ for the linear case of equation (22). If $u_{0}(z)$ satisfies the point conditions $u\left(z_{j}\right)=b_{j}, j=0,1, \ldots, K^{\prime}$ in $(3)$, then the solution is also a solution of Problem $P$ for the equation (22). Otherwise we can find $K^{\prime}=2 K-2 N+$ $J^{\prime}+1$ linearly independent solutions $u_{k}(z)\left(k=1, \ldots, K^{\prime}\right)$ of Problem $Q$ for the homogeneous equation of (22) satisfying the boundary conditions

$$
\begin{aligned}
\operatorname{Re}\left[\overline{\lambda(z)} u_{k z}\right] & =0, \quad z \in \Gamma^{*} \\
u_{k}\left(z_{0}\right) & =0 \\
\left.\operatorname{Im}\left[\overline{\lambda(z)} u_{k z}\right]\right|_{z=z_{j}^{\prime}} & =\delta_{j k}, \quad j=1, \ldots, K^{\prime} .
\end{aligned}
$$

It is easy to see that

$$
J=\left|\begin{array}{ccc}
u_{1}\left(z_{1}\right) & \ldots & u_{K^{\prime}}\left(z_{1}\right) \\
\vdots & \ddots & \vdots \\
u_{1}\left(z_{K^{\prime}}\right) & \ldots & u_{K^{\prime}}\left(z_{K^{\prime}}\right)
\end{array}\right| \neq 0
$$

Hence we can find $K^{\prime}$ real constants $d_{k}\left(k=1, \ldots, K^{\prime}\right)$, which are not all equal to 0 , such that $u(z)=u_{0}(z)+\sum_{k=1}^{K^{\prime}} d_{k} u_{k}(z)$ is a solution of Problem $P$ for $(22)$. Moreover, by using the method of parameter extension and the Schauder fixedpoint theorem, we can find a solution of Problem $P$ for the general equation (6) (see [6, Chap. III, Sec. 4]). Besides we can also prove the other part of the theorem.

In order to prove the existence of solutions of Problem $P$ for equation (1), we first introduce the nonlinear elliptic equation of second order

$$
u_{z \bar{z}}=F_{m}\left(z, u, u_{z}, u_{z z}\right), \quad F_{m}=\operatorname{Re}\left[Q_{m} u_{z z}+A_{1 m} u_{z}\right]+\hat{A}_{2 m} u+A_{3}
$$

in $D$, with the coefficients $\hat{A}_{2}=A_{2}+|u|^{\sigma}$ and

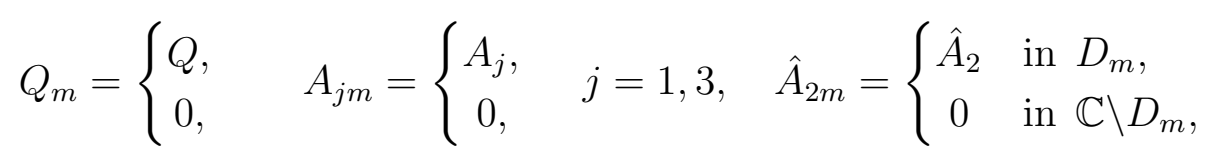

where $D_{m}=\left\{z \in D \mid \operatorname{dist}(z, \Gamma) \geq \frac{1}{m}\right\}, m$ is a positive integer.

Theorem 3.2. Let the equation (1) satisfy Conditions $C^{*}$, and $u(z)$ be any solution of Problem $P$ for equation (24). Then $u(z)$ can be expressed in the form

$$
u(z)=U(z)+\tilde{v}(z)=U(z)+\hat{v}(z)+v(z)
$$


in which $\tilde{v}(z)=\hat{v}(z)+v(z)$ is a solution of (24) with the homogeneous first boundary condition

$$
\tilde{v}(z)=0 \quad \text { on } \partial D_{0}=\{|z|=1\}
$$

where

$$
v(z)=H F_{m}=\frac{2}{\pi} \iint_{D_{0}} F_{m}\left(\zeta, u, u_{\zeta}, u_{\zeta \zeta}\right) \ln |z-\zeta| d \sigma_{\zeta},
$$

which is called Problem I. $U(z)$ is a solution of Problem I for $U_{z \bar{z}}=0$ in D satisfying the boundary condition (29) below, and $U(z), \tilde{v}(z)$ satisfy the estimates

$$
\hat{C}_{\delta}^{1}[U, \bar{D}]=C_{\delta}\left[|U|^{\sigma+1}, \bar{D}\right]+C_{\delta}\left[X(z) U_{z}, \bar{D}\right] \leq M_{6}, \quad \hat{C}_{\delta}^{1}\left[\tilde{v}, \overline{D_{0}}\right] \leq M_{7},
$$

where $\delta, M_{j}=M_{j}\left(\delta, k_{1}, p, D_{m}\right)(j=6,7)$ are non-negative constants.

Proof. It is clear that the solution $u(z)$ can be expressed as (25). On the basis of Theorem 2.3, it is easy to see that $U(z)$ satisfies the first estimate in (26), and then we know that $\tilde{v}(z)$ satisfies the second estimate of (26).

Theorem 3.3. If the equation (1) satisfies Conditions $C^{*}$, then Problem $P$ for the equation (24) has a solution.

Proof. We introduce the equation and the boundary condition with the parameter $t \in[0,1]$ :

$$
\begin{gathered}
u_{z \bar{z}}=t F_{m}\left(z, u, u_{z}, u_{z z}\right) \text { in } D_{0}, \\
\operatorname{Re}\left[\overline{\lambda(z)} u_{z}\right]-a_{2}(z)=-t a_{1}(z) u(z), \quad z \in \Gamma^{*} \\
u\left(z_{0}\right)=b_{0} \\
u\left(z_{j}\right)=b_{j}, \quad j=1, \ldots, K^{\prime} .
\end{gathered}
$$

Denote by $B_{M}$ a bounded open set in the Banach space $B=\hat{W}_{p_{0}}^{2}\left(D_{0}\right)=$ $\hat{C}_{\delta}^{1}\left(\overline{D_{0}}\right) \cap \tilde{W}_{p_{0}}^{2}\left(D_{0}\right)(0<\delta \leq \alpha)$, where $\hat{C}_{\delta}^{1}\left(\overline{D_{0}}\right)$ and $\tilde{W}_{p_{0}}^{2}\left(D_{0}\right)$ are as stated before, the elements of which are real functions $V(z)(\in B)$ satisfying the inequalities $\hat{C}_{\delta}^{1}\left[V, \overline{D_{0}}\right]<M_{8}=M_{7}+1$, in which $M_{7}$ is a non-negative constant as stated in (26). We choose any function $V(z) \in \overline{B_{M}}$ and substitute it into the appropriate position in the right hand side of (24). Afterwards we make an integral $v(z)=$ $H \rho$ as follows:

$$
v(z)=H \rho=\frac{2}{\pi} \iint_{D_{0}} \rho(\zeta) \log |z-\zeta| d \sigma_{\zeta},
$$

where $D_{0}=\{|z|<1\}, \rho(z)=V_{z \bar{z}}$. Next we find a solution $\hat{v}(z)$ of the boundary value problem in $D_{0}$ :

$$
\begin{aligned}
& \hat{v}_{z \bar{z}}=0 \quad \text { in } D_{0} \\
& \hat{v}(z)=-v(z) \text { on } \partial D_{0} \text {. }
\end{aligned}
$$


Denote $\tilde{v}(z)=v(z)+\hat{v}(z)$. Moreover by using a similar way as in [5] and [6], we can find a solution $U(z)$ of the boundary value problem in $D_{0}$ :

$$
U_{z \bar{z}}=0 \text { in } D
$$

and

$$
\left\{\begin{aligned}
\operatorname{Re}\left[\overline{\lambda(z)}(U+\tilde{v})_{z}\right]+t a_{1}(z)(U+\tilde{v}) & =a_{2}(z) & & \text { on } \Gamma^{*} \\
U\left(z_{0}\right)+\tilde{v}\left(z_{0}\right) & =b_{0} & & \\
U\left(z_{j}\right)+\tilde{v}\left(z_{j}\right) & =b_{j}, & & j=1, \ldots, K^{\prime} .
\end{aligned}\right.
$$

Now we discuss the equation

$$
\tilde{V}_{z \bar{z}}=t F_{m}\left(z, u, u_{z}, U_{z z}+\tilde{v}_{z z}\right), \quad 0 \leq t \leq 1,
$$

and boundary condition

$$
\tilde{V}(z)=0 \quad \text { on } \quad \partial D_{0}, \quad 0 \leq t \leq 1,
$$

where $u(z)=U(z)+\tilde{v}(z)$. By Conditions $C^{*}$ and the principle of contracting mappings, the above Problem $I$ for the equation (30) in $D_{0}$ has a unique solution $\tilde{V}(z)$. Denote by $\tilde{V}=S(V, t)(0 \leq t \leq 1)$ the mapping from $V$ onto $\tilde{V}$. Furthermore, if $u(z)$ is a solution of Problem $P$ in $D_{0}$ for the equation

$$
u_{z \bar{z}}=t F_{m}\left(z, u, u_{z}, u_{z z}\right), \quad 0 \leq t \leq 1,
$$

and boundary conditions

$$
\left\{\begin{aligned}
\operatorname{Re}\left[\overline{\lambda(z)} u_{z}\right]-a_{2}(z) & =-t a_{1}(z) u(z) \text { on } \Gamma^{*} \\
u\left(z_{0}\right) & =b_{0}, \\
u\left(z_{j}\right) & =b_{j}, \quad j=1, \ldots, K^{\prime}, \quad 0 \leq t \leq 1,
\end{aligned}\right.
$$

then it follows from Theorem 2.3, that the solution $u(z)$ of Problem $P$ for (31) satisfies (10). Consequently $\tilde{V}(z)=u(z)-U(z) \in B_{M}$. Set $B_{0}=B_{M} \times[0,1]$. In the following, we shall verify that the mapping $\tilde{V}=S(V, t)$ satisfies the three conditions of Leray-Schauder theorem:

1. For every $t \in[0,1], \tilde{V}=S(V, t)$ continuously maps the Banach space $B$ into itself, and it is completely continuous in $B_{M}$. Besides, for every function $V(z) \in \overline{B_{M}}, S(V, t)$ is uniformly continuous with respect to $t \in[0,1]$.

In fact, we arbitrarily choose $V_{n}(z) \in \overline{B_{M}}, n=1,2, \ldots$, it is clear that from $\left\{V_{n}(z)\right\}$ there exists a subsequence $\left\{V_{n_{k}}(z)\right\}$, such that $\left\{V_{n_{k}}(z)\right\},\left\{V_{n_{k} z}(z)\right\}$ and corresponding functions $\left\{U_{n_{k}}(z)\right\},\left\{U_{n_{k}}(z)\right\}$ uniformly converge to $V_{0}(z)$, $V_{0 z}(z), U_{0}(z), U_{0 z}(z)$ in $\bar{D}_{0}, \bar{D}$, respectively. We can find a solution $\tilde{V}_{0}(z)$ of Problem $D$ for the equation $\tilde{V}_{0 z \bar{z}}=t F_{m}\left(z, u_{0}, u_{0 z}, U_{0 z z}+\tilde{v}_{0 z z}\right), 0 \leq t \leq 1$, 
where $u_{0}(z)=U_{0}(z)+v_{0}(z)+\hat{v}_{0}(z)=S_{1}\left(V_{0}, t\right)$. From $\tilde{V}_{n_{k}}=S\left(V_{n_{k}}, t\right)$ and $\tilde{V}_{0}=S\left(V_{0}, t\right)$, we have

$$
\begin{aligned}
\left(\tilde{V}_{n_{k}}-\tilde{V}_{0}\right)_{z \bar{z}}= & t\left[F_{m}\left(z, u_{n_{k}}, u_{n_{k} z}, U_{n_{k} z z}+\tilde{v}_{n_{k} z z}\right)\right. \\
& \left.-F_{m}\left(z, u_{n_{k}}, u_{n_{k} z}, U_{n_{k} z z}+\tilde{v}_{0 z z}\right)+C_{n_{k}}(z)\right], \quad 0 \leq t \leq 1,
\end{aligned}
$$

where $C_{n_{k}}=F_{m}\left(z, u_{n_{k}}, u_{n_{k} z}, U_{n_{k} z z}+\tilde{v}_{0 z z}\right)-F_{m}\left(z, u_{0}, u_{0}, U_{0 z z}+\tilde{v}_{0 z z}\right)$ in $D_{0}$. By Theorem 2.3 and the conditions of equation (24), we can verify that

$$
L_{2}\left[C_{n_{k}}, \overline{D_{0}}\right] \rightarrow 0 \quad \text { as } k \rightarrow \infty .
$$

In fact, by Conditions $C^{*}$ and the conditions of equation (24), it is not difficult to see that $C_{n_{k}}$ converges to 0 for almost every point $z \in D_{0}$. Thus for two arbitrary sufficiently small positive constants $\varepsilon_{1}$ and $\varepsilon_{2}$, there exists a subset $D_{*}$ in $D_{0}$ and a positive integer $N$, so that meas $D_{*}<\varepsilon_{1}$ and $\left|C_{n_{k}}\right|<\varepsilon_{2}$ in $\bar{D}_{0} \backslash D_{*}$ for $n>N$. By the Hölder inequality and the Minkowski inequality, we have

$$
\begin{aligned}
L_{p_{0}}\left[C_{n_{k}}, \overline{D_{0}}\right] & \leq L_{p_{0}}\left[C_{n_{k}}, D_{*}\right]+L_{p_{0}}\left[C_{n_{k}}, \bar{D}_{0} \backslash D_{*}\right] \\
& \leq L_{p_{1}}\left[C_{n_{k}}, D_{*}\right] L_{p_{2}}\left[1, D_{*}\right]+\varepsilon_{2}(\operatorname{meas} D)^{\frac{1}{p_{0}}} \\
& \leq L_{p_{1}}\left[C_{n_{k}}, D_{*}\right] \varepsilon_{1}^{\frac{1}{p_{2}}}+\varepsilon_{2} \pi^{\frac{1}{p_{0}}} \\
& \leq M_{0} \varepsilon_{1}^{\frac{1}{p_{2}}}+\varepsilon_{2} \pi^{\frac{1}{p_{0}}}=\varepsilon
\end{aligned}
$$

in which $M_{0}=\sup _{n_{k}} L_{p_{1}}\left[C_{n_{k}}, D_{*}\right], p_{1}$ is a constant such that $p_{0}<p_{1}<\min (p$, $\left.\frac{1}{1-\alpha}\right)$ and $p_{2}=\frac{p_{0} p_{1}}{p_{1}-p_{0}}$. Therefore the formula (32) is true. Similarly to (10)-(20), we can derive that

$$
\left\|\tilde{V}_{n_{k}}-\tilde{V}_{0}\right\|_{\tilde{W}_{p_{0}}^{2}\left(D_{0}\right)} \leq \frac{L_{2}\left[C_{n_{k}}, \overline{D_{0}}\right]}{\left[1-q_{0}\right]},
$$

where $q_{0}<1$. It is not difficult to show that $\left\|\tilde{V}_{n_{k}}-\tilde{V}_{0}\right\|_{\tilde{W}_{p_{0}}^{2}\left(D_{0}\right)} \rightarrow 0$ as $k \rightarrow \infty$. Moreover, from Theorem 2.3, we can verify that there exists a subsequence of $\left\{\tilde{V}_{n_{k}}(z)-\tilde{V}_{0}(z)\right\}$, denoted by $\left\{\tilde{V}_{n_{k}}(z)-\tilde{V}_{0}(z)\right\}$ again, such that $\hat{C}_{\delta}^{1}\left[\tilde{V}_{n_{k}}-\tilde{V}_{0}, \overline{D_{0}}\right] \rightarrow$ 0 as $k \rightarrow \infty$. This shows the complete continuity of $\tilde{V}=S(V, t)(0 \leq t \leq 1)$ in $\overline{B_{M}}$. By using a similar method, we can prove that $\tilde{V}=S(V, t)(0 \leq t \leq 1)$ continuously maps $\overline{B_{M}}$ into $B$, and $\tilde{V}=S(V, t)$ is uniformly continuous with respect to $t \in[0,1]$ for $V \in \overline{B_{M}}$.

2. For $t=0$, from Theorem 2.3 and the estimates in (26), it is clear that $\tilde{V}=S(V, 0) \in B_{M}$.

3. From Theorem 2.3 and (26), we see that $\tilde{V}=S(V, t)(0 \leq t \leq 1)$ has no solution $V(z)$ on the boundary $\partial B_{M}=\overline{B_{M}} \backslash B_{M}$.

Hence by the Leray-Schauder theorem, we know that Problem $P$ for the equation (27) and boundary condition (28) with $t=1$, namely (24) has a solution $u(z)=U(z)+\tilde{V}(z)=U(z)+v(z)+\hat{v}(z)=S_{1}(V, 1) \in B_{M}$. 
Theorem 3.4. Under the same conditions as in Theorem 2.3, Problem P for the equation (1) has a solution.

Proof. By Theorem 2.3 and Theorem 3.2, Problem $P$ for the equation (24) possesses a solution $u_{m}(z)$, and the solution $u_{m}(z)$ of Problem $P$ for $(24)$ satisfies the estimate $(10)$, where $m=1,2, \ldots$ Thus, we can choose a subsequence $\left\{u_{m_{k}}(z)\right\}$, such that $\left\{u_{m_{k}}(z)\right\},\left\{u_{m_{k} z}(z)\right\}$ in $\bar{D}$ uniformly converge to $u_{0}(z), u_{0 z}(z)$, respectively. Obviously, $u_{0}(z)$ satisfies the boundary condition $(2)$ of Problem $P$. Finally we can verify that $u_{0}(z)$ is a solution of equation (1) (see [6, Chap. III, Sec. 3]).

Remark 3.5. In the above discussion, it is assumed that $K \geq N-\frac{1}{2}$. Actually the index $K$ can be equal to $N-1$. In this case the boundary condition (2) should be replaced by

$$
\frac{\partial u}{\partial \nu}+2 a_{1}(z) u(z)=2 a_{2}(z)+h(z), \quad z \in \Gamma^{*}, \quad u\left(z_{0}\right)=b_{0},
$$

where $h(z)=0$ on $\Gamma_{k}^{\prime}, k=1, \ldots, m$, and $h(z)=h_{0}$ on $\Gamma_{m+1}^{\prime}, h_{0}$ is an undetermined real constant. As stated in the proof of Theorem 2.1, if the positive maximum $M$ of $U(z)$ is taken at a point $z^{*} \in \Gamma_{m+1}^{\prime}$, then $\frac{\partial U}{\partial \nu}+2 a_{1}^{*}(z) U(z)=h_{0} \geq 0$; and if the negative minimum $m$ of $U(z)$ is taken at a point $z_{*} \in \Gamma_{m+1}^{\prime}$, then $\frac{\partial U}{\partial \nu}+2 a_{1}^{*}(z) U(z)=h_{0} \leq 0$, thus $h_{0}=0$. Hence $U(z)$ cannot attain its positive maximum and negative minimum on $\Gamma_{m+1}^{\prime}$. This shows that the uniqueness theorem for $K=N-1$ is also valid. Therefore the above theorems for $K=N-1$ are true. The case $K<N-1$ remains to be further discussed.

Remark 3.6. Finally we explain that the continuous irregular oblique derivative problem is a special case of the above Problem $P$, namely we find the continuously differentiable solution $u(z)$ of equation (1) in $\bar{D}$ satisfying the boundary conditions

$$
\frac{\partial u}{\partial \nu}+2 a_{1}(z) u(z)=2 a_{2}(z), \quad z \in \Gamma, u\left(z_{j}\right)=b_{j}, \quad j=0,1, \ldots, K^{\prime},
$$

where $\nu\left(=\nu_{1}+i \nu_{2} \neq 0\right)$ can be arbitrary at every point on $\Gamma, K^{\prime}(=2 K-$ $2 N+J+1 \geq 0), J$ are non-negative integers as stated below. It is clear that the boundary condition (33) can be rewritten in the complex form

$$
\operatorname{Re}\left[\overline{\lambda(z)} u_{z}\right]+a_{1}(z) u=a_{2}(z), \quad z \in \Gamma, \quad u\left(z_{j}\right)=b_{j}, \quad j=0,1, \ldots, K^{\prime},
$$

in which $\lambda(z)=\cos (\nu, x)+i \sin (\nu, x)=e^{i(\nu, x)} \neq 0,(\nu, x)$ is the angle between $\nu$ and the $x$-axis, $z_{j}\left(\in \Gamma_{j}, j=0,1, \ldots, K^{\prime}\right)$ are distinct points on $\Gamma$. Suppose that $\lambda(z), a_{1}(z), a_{2}(z)$ satisfy the conditions

$$
\begin{array}{rlrl}
C_{\alpha}[\lambda(z), \Gamma] & \leq k_{0}, & \left|b_{j}\right| & \leq k_{2}, \quad j=0,1, \ldots, K^{\prime}, \\
C_{\alpha}\left[a_{1}(z), \Gamma\right] & \leq k_{0}, & 0 \leq a_{1}(z) \cos (\nu, n) \text { on } \Gamma, \\
C_{\alpha}\left[a_{2}(z), \Gamma\right] & \leq k_{2}, & &
\end{array}
$$


in which $\alpha\left(\frac{1}{2}<\alpha<1\right), k_{2}$ are non-negative constants. The boundary $\partial D=\Gamma$ can be divided into two parts, namely $E^{+} \subset\left\{z \in \partial D \mid \cos (\nu, n) \geq 0, a_{1} \geq 0\right\}$ and $E^{-} \subset\left\{z \in \partial D \mid \cos (\nu, n) \leq 0, a_{1} \leq 0\right\}$, and $E^{+} \cap E^{-}=\emptyset, E^{+} \cup E^{-}=\Gamma$, $\overline{E^{+}} \cap \overline{E^{-}}=E^{0}$. For every component $L=\Gamma_{j}(0 \leq j \leq N)$ of $\Gamma$, there are three cases: 1. $L \subset E^{+}$. 2. $L \subset E^{-}$. In these cases, if $\cos (\nu, n) \equiv 0, a_{1}(z) \equiv 0$ on $\Gamma_{j}$ $(1 \leq j \leq J, J \leq N+1)$, and the above identical formulas on $\Gamma_{j}(J<j \leq N+1)$ do not hold, then we need the conditions $\int_{\Gamma_{j}} a_{2}(z) d s=0(1 \leq j \leq J)$, and $u\left(z_{j}\right)=b_{j}, j=0,1, \ldots, K^{\prime}(\geq J)$, in which $z_{j}, b_{j}\left(j=0,1, \ldots, K^{\prime}\right)$ are as stated before, and denote $\Gamma^{\prime}=\bigcup_{j=1}^{J} \Gamma_{j}, \Gamma^{\prime \prime}=\bigcup_{j=J+1}^{N+1} \Gamma_{j}$. 3. There exists at least a point on each component of $L^{+}=E^{+} \cap L$ and $L^{-}=E^{-} \cap L$, such that $\cos (\nu, n) \neq 0$ at the point, and $E^{0} \cap L \in\left\{z_{0}, z_{1}, \ldots, z_{K^{\prime}}\right\}$ such that every component of $L^{+}, L^{-}$includes its initial point and does not include its terminal point; and $z_{j} \in \overline{L^{+}} \cap L^{-}\left(0 \leq j \leq K^{\prime}\right)$, when the direction of $\nu$ at $z_{j}$ is equal to the direction of $L$; and $z_{j} \in L^{+} \cap \overline{L^{-}}\left(0 \leq j \leq K^{\prime}\right)$, when the direction of $\nu$ at $z_{j}$ is opposite to the direction of $L$; and $\cos (\nu, n)$ changes the sign once on the two components with the end point $z_{j}\left(0 \leq j \leq K^{\prime}\right)$; we may assume that $u\left(z_{j}\right)=b_{j}, j=0,1, \ldots, K^{\prime}$. The above boundary value problem for $(1)$ is called Problem $P^{\prime}$, and Problem $P^{\prime}$ with homogeneous boundary condition of (33) or (34) is called Problem $P_{0}^{\prime}$. The number $K=\frac{1}{2}\left(K_{1}+\cdots+K_{N+1}\right)$, where $K_{j}=\Delta_{\Gamma_{j}} \arg \lambda(z), j=1, \ldots, N+1$, is called the index of Problem $P^{\prime}$. We can choose $K^{\prime}=2 K-2 N+J+1$. As a special case of Problem $P$ in Sections 1-3, we see that Problem $P^{\prime}$ for the equation (1) has a solution.

\section{References}

[1] Begehr, H. and G. C. Wen: Nonlinear Elliptic Boundary Value Problems and Their Applications. Harlow: Longman 1996.

[2] Begehr, H. and G. C. Wen: The discontinuous oblique derivative problem for nonlinear elliptic systems of first order. Rev. Roumanie Math. Pures Appl., 33 (1988), 7-19.

[3] Monakhov, V. N.: Boundary Value Problem with Free Boundaries for Elliptic Systems of Equations. Translations of Mathematics Monographs 57. Providence (RI): Amer. Math. Soc. 1983.

[4] Vekua, I. N.: Generalized Analytic Functions. Oxford: Pergamon 1962.

[5] Wen, G. C.: Conformal Mappings and Boundary Value Problems. Translations of Mathematics Monographs 106. Providence (RI): Amer. Math. Soc. 1992.

[6] Wen, G. C. and H. Begehr: Boundary Value Problems for Elliptic Equations and Systems, Harlow: Longman 1990.

[7] Wen, G. C.: Approximate Methods and Numerical Analysis for Elliptic Complex Equations. Amsterdam: Gordon and Breach 1999.

Received March 10, 2006; revised September 15, 2006 